\title{
Widespread Hypomethylation Occurs Early and Synergizes with Gene Amplification during Esophageal Carcinogenesis
}

\author{
Hector Alvarez ${ }^{1}$, Joanna Opalinska ${ }^{29}$, Li Zhou ${ }^{29}$, Davendra Sohal ${ }^{2}$, Melissa J. Fazzari ${ }^{3}$, Yiting $\mathbf{Y u}^{2}$, \\ Christina Montagna ${ }^{2}$, Elizabeth A. Montgomery ${ }^{1}$, Marcia Canto ${ }^{4}$, Kerry B. Dunbar ${ }^{4}$, Jean Wang ${ }^{4}$, Juan \\ Carlos Roa ${ }^{5}$, Yongkai Mo $^{2}$, Tushar Bhagat ${ }^{2}$, K. H. Ramesh ${ }^{6}$, Linda Cannizzaro ${ }^{2}$, J. Mollenhauer ${ }^{7}$, Reid F. \\ Thompson $^{2}$, Masako Suzuki ${ }^{2}$, Stephen Meltzer ${ }^{4}$, Ari Melnick ${ }^{8}$, John M. Greally ${ }^{2,9{ }^{*}} *$, Anirban \\ Maitra ${ }^{1,10,111^{*}}$, Amit Verma ${ }^{2 \Phi_{*}}$
}

1 Department of Pathology, Johns Hopkins University School of Medicine, Baltimore, Maryland, United States of America, 2 Department of Medicine, Albert Einstein College of Medicine, Bronx, New York, United States of America, 3 Department of Epidemiology and Population Health, Albert Einstein College of Medicine, Bronx, New York, United States of America, 4 Department of Medicine, Johns Hopkins University School of Medicine, Baltimore, Maryland, United States of America, 5 Department of Pathology, Universidad de la Frontera, Temuco, Chile, 6 Department of Pathology, Albert Einstein College of Medicine, Bronx, New York, United States of America, 7 Molecular Oncology, Medical Biotechnology Center, University of Southern Denmark, Odense, Denmark, 8 Weil Cornell College of Medicine, New York, New York, United States of America, 9 Department of Genetics, Albert Einstein College of Medicine, Bronx, New York, United States of America, 10 Department of Oncology, Johns Hopkins University School of Medicine, Baltimore, Maryland, United States of America, 11 McKusick-Nathans Institute of Genetic Medicine, Johns Hopkins University School of Medicine, Baltimore, Maryland, United States of America

\begin{abstract}
Although a combination of genomic and epigenetic alterations are implicated in the multistep transformation of normal squamous esophageal epithelium to Barrett esophagus, dysplasia, and adenocarcinoma, the combinatorial effect of these changes is unknown. By integrating genome-wide DNA methylation, copy number, and transcriptomic datasets obtained from endoscopic biopsies of neoplastic progression within the same individual, we are uniquely able to define the molecular events associated progression of Barrett esophagus. We find that the previously reported global hypomethylation phenomenon in cancer has its origins at the earliest stages of epithelial carcinogenesis. Promoter hypomethylation synergizes with gene amplification and leads to significant upregulation of a chr4q21 chemokine cluster and other transcripts during Barrett neoplasia. In contrast, gene-specific hypermethylation is observed at a restricted number of loci and, in combination with hemi-allelic deletions, leads to downregulatation of selected transcripts during multistep progression. We also observe that epigenetic regulation during epithelial carcinogenesis is not restricted to traditionally defined "CpG islands," but may also occur through a mechanism of differential methylation outside of these regions. Finally, validation of novel upregulated targets (CXCL1 and 3, GATA6, and DMBT1) in a larger independent panel of samples confirms the utility of integrative analysis in cancer biomarker discovery.
\end{abstract}

Citation: Alvarez H, Opalinska J, Zhou L, Sohal D, Fazzari MJ, et al. (2011) Widespread Hypomethylation Occurs Early and Synergizes with Gene Amplification during Esophageal Carcinogenesis. PLoS Genet 7(3): e1001356. doi:10.1371/journal.pgen.1001356

Editor: James M. Ford, Stanford University School of Medicine, United States of America

Received June 14, 2010; Accepted February 25, 2011; Published March 31, 2011

Copyright: (C) 2011 Alvarez et al. This is an open-access article distributed under the terms of the Creative Commons Attribution License, which permits unrestricted use, distribution, and reproduction in any medium, provided the original author and source are credited.

Funding: This work was supported by NIH R01HL082946, Gabrielle Angel Foundation, Hershaft Family Foundation, Leukemia and Lymphoma Society Partnership for Cures grant, American Cancer Society, and Immunooncology Training Program T32 CA009173. The funders had no role in study design, data collection and analysis, decision to publish, or preparation of the manuscript.

Competing Interests: The authors have declared that no competing interests exist.

*E-mail: john.greally@einstein.yu.edu (JM Greally); amaitra1@jhmi.edu (A Maitra); averma@aecom.yu.edu (A Verma)

9 These authors contributed equally to this work.

- These authors also contributed equally to this work.

\section{Introduction}

The incidence of esophageal adenocarcinoma (EAG) is increasing at an alarming pace in the United States $(>600 \%$ increase since 1975) [1]. Since most patients with EAC present at diagnosis with an advanced disease stage, the 5-year survival rate is a dismal $13 \%$ [2], underscoring the pressing need for early diagnostic biomarkers, as well as for improved therapeutic strategies, in this malignancy. Distinct pathological stages of specialized columnar epithelium (Barrett metaplasia) and low- followed by high-grade dysplasia precede adenocarcinoma [3,4]. Barrett esophagus (BE) is defined as a change in the esophageal epithelium that can be recognized grossly by a distinct salmon pink color at endoscopy, and confirmed by the presence of specialized columnar epithelium on biopsy. The prevalence of $\mathrm{BE}$ is not precisely known, but it has been estimated to range between $1-10 \%$ of the general population [5]. The incidence of EAC in patients with $\mathrm{BE}$ is increased 100-fold above that of the general population [6]. Thus, BE, with or without associated epithelial dysplasia, provides a unique opportunity for risk stratification and secondary prevention of EAC.

Expression profiling of $\mathrm{BE}$ and $\mathrm{EAC}$ using genome-wide approaches has identified many of the transcriptomic alterations 


\section{Author Summary}

The incidence of esophageal adenocarcinoma (EA) is increasing at an alarming pace in the United States. Distinct pathological stages of Barrett's metaplasia and low- and high-grade dysplasia can be seen preceding malignant transformation. These precursor lesions provide a unique in vivo model for deepening our understanding the early steps in human neoplasia. By integrating genome-wide DNA methylation, copy number, and transcriptomic datasets obtained from endoscopic biopsies of neoplastic progression within the same individual, we are uniquely able to define the molecular events associated progression of Barrett esophagus. We show that the predominant change during this process is loss of DNA methylation. We show that this global hypomethylation occurs very early during the process and is seen even in preinvasive lesions. This loss of DNA methylation drives carcinogenesis by cooperating with gene amplifications in upregulating proteins during this process. Finally we uncovered proteins that upregulated by loss of methylation or gene amplification (CXCL1 and 3, GATA6, and DMBT1) and show their relevance by validating their levels in larger independent panel of samples, thus confirming the utility of integrative analysis in cancer biomarker discovery.

occurring during esophageal neoplastic progression [7-10]. In several instances, it has also been possible to identify the proximate genomic or epigenetic mechanism (for example, intragenic deletion, truncating mutation, copy number aberration, or promoter methylation, respectively) contributing to the altered expression [11-16]. This strategy has unequivocally yielded a rich seedbed of candidate biomarkers for diagnosis, as well as for prognostication, of BE neoplasia [17-20]. Nonetheless, there remains a notable lacuna in globally integrating transcriptomic abnormalities during Barrett progression with the corresponding changes occurring at the level of the genome and epigenome. We reasoned that a multi-platform integrated approach would not only enable the elucidation of novel biomarkers, but also clarify the genomic and/or epigenetic mechanisms driving transcript abnormalities during carcinogenesis. Such integration of global datasets has begun to emerge in solid tumors [21,22], but to the best of our knowledge this has not been performed in precursor lesions, especially in the context of multistep progression occurring in a single individual. Herein, we provide an unbiased and comprehensive approach for integrating large-scale genomic, epigenetic, and transcriptomic datasets, obtained using tissue from patients undergoing endoscopic mucosal biopsy for BE. In contrast to numerous prior studies using two platforms (for example, combined copy number and expression analysis) where a "hit" on the second allele is typically inferred, the multi-platform analysis performed here can directly confirm or refute the Knudsonian paradigm for a given altered transcript. Our studies have identified striking epigenomic alterations, and in specific, widespread hypomethylation, which occurs at the earliest stages of epithelial carcinogenesis. This approach is in direct contrast to most singleor limited-locus studies that have focused on epigenetic silencing of candidate tumor suppressor genes by hypermethylation of the promoter. In addition, we have identified clustered transcripts, such as the chemokine ligands CXCL1 and 3, that are markedly upregulated via simultaneous biallelic alteration by hypomethylation and gene amplification, respectively, and whose protein products have the potential to serve as serum biomarkers of neoplastic progression in $\mathrm{BE}$.

\section{Results}

Widespread hypomethylation is an early event in the progression of Barrett esophagus

Eight histologically validated endoscopic mucosal biopsies representing normal squamous mucosa and the various histopathological stages of Barrett esophagus progression were obtained from three patients undergoing repeat endoscopy for dysplasia (Figure 1A, Figure S1); two additional unmatched gastric cardia biopsies were obtained as controls for the intended profiling studies. Prospective as well as retrospective surveillance studies have convincingly established that nondysplastic BE and lowgrade dysplasia (LGD) both have a significantly lower risk of progression to EAC, when compared to high-grade dysplasia (HGD); in fact, for the purposes of therapeutic decision-making between continued surveillance versus local ablation, stratification typically occurs based on the diagnosis of HGD in BE mucosa. Therefore, with the objective of pair-wise comparison, samples of non-dysplastic BE and LGD were categorized as "low", while the two HGD samples and one EAC were categorized as "high". Nucleic acids extracted from cryostat-embedded sections of the ten samples were utilized for three concurrent microarray-based assays: 1) gene expression profiling, 2) array comparative genomic hybridization (aCGH), and 3) genome-wide cytosine methylation, thus simultaneously querying the transcriptome, genome, and epigenome, respectively. Methylation profiling was performed using the $\underline{H} p a \mathrm{II}$ tiny fragment Enrichment by Ligation-mediated PCR (HELP) assay, which compares HpaII (methylation-sensitive) and $M s p \mathrm{I}$ (methylation insensitive) genomic representations to identify hypo- and hypermethylated loci in the genome.[23-25]. The HELP HpaII / MspI ratios were validated at over 60 independent loci by mass spectrometry-based high-throughput quantitative methylation PCR analysis (Sequenom EpiTYPER); based on this quantitative approach, a HpaII/MspI ratio of 0.3 corresponded to $50 \%$ cytosine methylation (Figure S2) and was used as a threshold for defining hypo- or hypermethylation.

Unsupervised hierarchical clustering analyses demonstrated that, at the level of the transcriptome, squamous mucosa clustered discretely from "glandular" epithelium (including gastric cardiac as well as all stages of BE progression: Figure 1B); in contrast, at the level of the epigenome, "normal" mucosa (including both squamous and cardiac subtypes) clustered discretely from all "abnormal" (i.e., BE) epithelia (Figure 1C). These results suggest some degree of commonality of epigenetic profiles between otherwise normal gastrointestinal tissues, despite obvious morphological differences. A pair-wise comparison of transcriptomic profiles between normal esophageal squamous and gastric cardiac mucosal samples revealed large numbers of significantly differentially expressed transcripts, consistent with the distinct histogenesis and biologies of these normal mucosal subtypes (Figure 1D, left); in contrast, global cytosine methylation profiles between the two mucosal locations were considerably more overlapping, with significant differences in either hypo- or hypermethylation restricted to fewer loci (Figure 1E, left).

In pair-wise comparisons of gene expression during $\mathrm{BE}$ progression, we found large numbers of significantly differentially expressed transcripts between the early lesions of Barrett metaplasia and LGD (both classified as "Low") versus normal squamous mucosa, confirming the previous observation [7] that even non-dysplastic Barrett epithelium may harbor profound transcriptomic aberrations, some comparable to EAC (Figure 1D, middle). Significant gene expression differences between "high and "low" BE lesions were more attenuated and restricted to only a handful of loci (Figure 1D, right). While these gene expression 
A

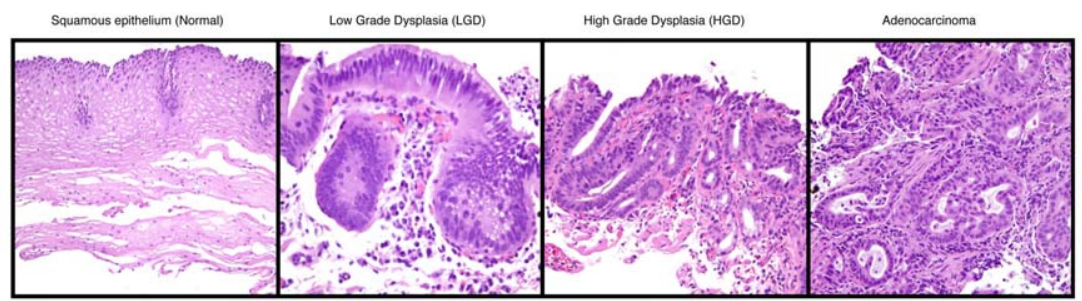

B

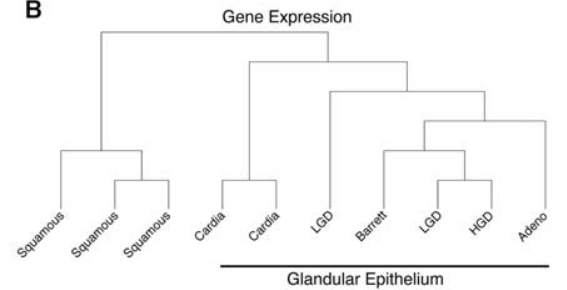

C
D

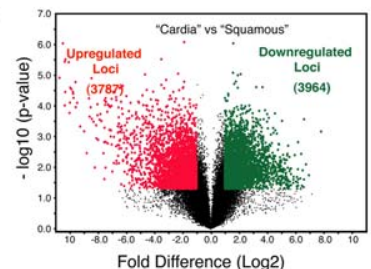

Fold Difference (Log2)

$\mathbf{E}$

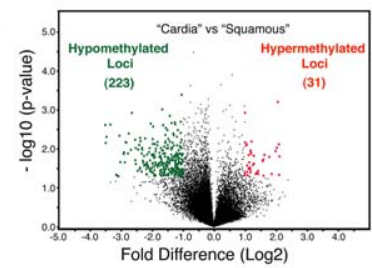

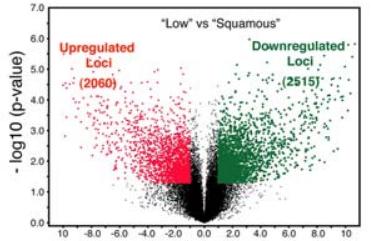

Fold Difference (Log2)

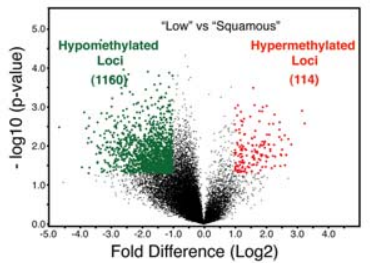

Methylation
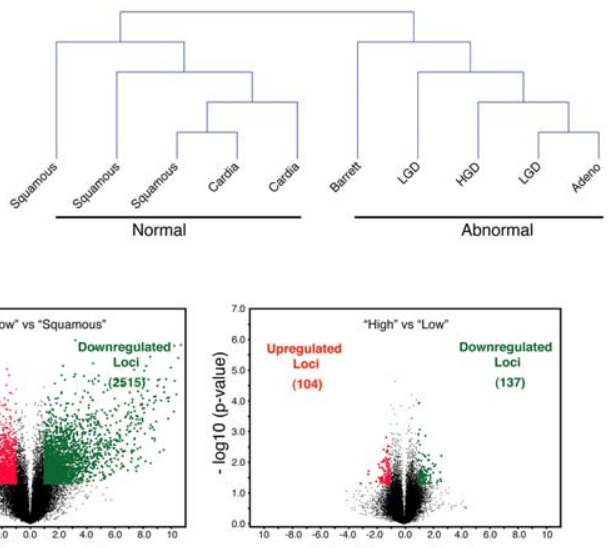

Fold Difference (Log2)

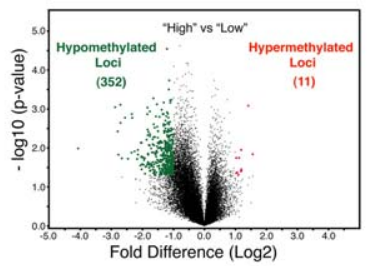

$\mathbf{F}$
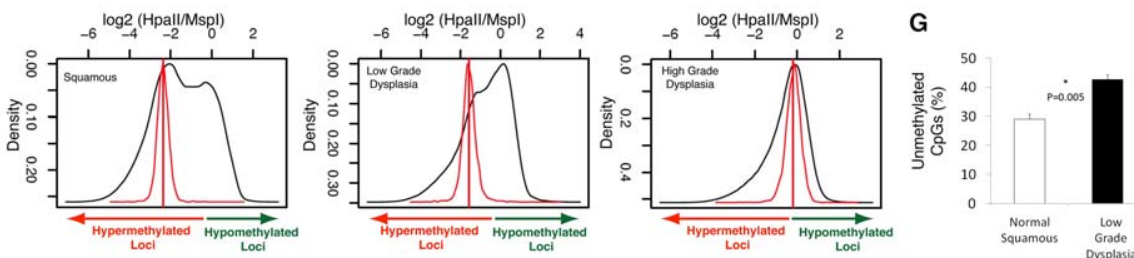

Figure 1. Global hypomethylation is seen early during esophageal carcinogenesis. Multiple endoscopic biopsies collected at the same time from patients with clinical history of Barrett's esophagus, were classified as normal squamous epithelium, Barrett's metaplasia, low grade dysplasia (LGD), high grade dysplasia (HGD) and adenocracinoma based on histology. (Panel A). Frozen tissue samples were homogenized, used for DNA and RNA isolation and were subjected to global expression profiling, DNA methylation profiling and DNA copy number analysis. Unsupervised hierarchical clustering based on gene expression profiling shows clustering of distinct histologic tissues with glandular tissue from stomach cardia being more similar to metaplastic and dysplastic tissues (Panel B). Clustering based on methylation shows dissimilarity between normal (esophageal and stomach) and abnormal tissues (Panel C). Volcano plots demonstrating significance and magnitude of change in mean gene expression (Panel D) and DNA methylation (Panel E) are shown. Each plot represents either 36,847 genes from the microarray experiment (D) or 25,626 Hpall-amplifiable fragments (HAF) from the HELP assay (E). The $\log _{2}$ ratio of difference of means is shown on the $x$-axis and the negative log of the $p$-value (based on paired T-Test) is shown on the y-axis. Genes that are significantly downregulated (panel D) or hypermethylated (panel E) are shown in green and red dots indicate genes that are either upregulated (panel D) or hypomethylated (panel E). Global methylation is shown by density plots of log ${ }_{2}$ of Hpall/ Mspl ratios and shows bimodal distribution in normal epithelial tissues with both hypo and hypermethylated loci. The red line denotes the median Hpall/Mspl ratio for random probes. A shift towards hypomethylation is observed during transformation of normal to low grade dysplasia in a representative series of samples (Panel F). Quantitative methylation for matched pairs of normal and Barrett's dysplasia as done by the LUMA assay. $X$ axis shows mean percent hypomethylation of 3 independent paired samples. (Panel $G$ ).

doi:10.1371/journal.pgen.1001356.g001

data were confirmatory of published results, methylation profiling revealed an unexpected dimension to epigenetic dysregulation during BE progression. Contrary to the hypermethylation reported in previous single-locus studies, we identified significant hypomethylation occurring at a large number of loci genome-wide during the transition of squamous mucosa to Barrett epithelium (1160 hypomethylated versus 114 hypermethylated loci., Figure 1E, middle,); since this epigenetic "shift" is not observed in the comparison of normal esophageal squamous versus cardiac mucosal samples, we believe these methylation alterations may be reflective 
of the actual BE disease process, rather than simply due to acquisition of columnar histology. A second, smaller wave of hypomethylation was observed when comparing "high" versus "low" BE categories (Figure 1E, right). This progressive hypomethylation was seen in methylation profiles of samples from the same patients (Figure 1F). Validation at the whole-genome level by the Luminometric methylation assay (LUMA) revealed a significantly large increase in unmethylated CpGs in "low" BE samples versus matched normal squamous mucosa (Figure 1G). These results demonstrate that the previously reported global hypomethylation observed in human cancers [26] can initiate at a very early stage of neoplastic transformation, such as in the noninvasive precursors of EAC.

\section{Differential methylation within gene promoters is not restricted to $\mathrm{CpG}$ islands but also involves CG dinucleotides outside of these islands}

In addition to this panoramic view of epigenetic shifts, we also assessed the nature of the HpaII sites showing altered methylation during BE progression. As our microarray design includes both canonical CpG islands and additional CG dinucleotide loci within gene promoters, we sought to test whether CpG islands, long considered the principal target of epigenetic dysregulation in cancer [27], were disproportionately affected. We compared the proportions of loci at which differential methylation was occurring within CpG islands versus other CG dinucleotide loci represented on the microarray. We determined that the majority of HpaII loci exhibiting differential methylation during $\mathrm{BE}$ progression lay, paradoxically, outside of canonical CpG islands (Figure 2A). To further validate this observation at a representative locus, we chose the example of Deleted in Malignant Brain Tumor 1 (DMBT1), whose gene promoter lacks a defined $\mathrm{CpG}$ island, yet demonstrates progressive hypomethylation accompanied by significant transcript upregulation during BE progression (Figure 2B). Histological examination of DMBT1 protein expression in a large archival cohort of $120 \mathrm{BE}$ samples and 54 normal controls revealed significant $(P<0.05)$ upregulation of this protein early during $\mathrm{BE}$ neoplasia (Figure 2C, 2D), thus validating the results obtained from our array-based analysis. These results suggest an epigenetic regulatory function for $\mathrm{CG}$ dinucleotide elements in the genome that do not meet the threshold for canonical CpG islands, which are strictly defined on base compositional criteria [28].

\section{Integrated multi-platform analysis provides experimental evidence of silencing of tumor suppressor genes by both genetic and epigenetic mechanisms in concordance with Knudson's two hit hypothesis}

Subsequently, in order to generate a multi-component genetic model of BE progression, we developed an in silico algorithm for integrating data from these three high-resolution platforms (i.e., gene expression, HELP, and aCGH), using genomic coordinates for the respective probes from each of the platform arrays (Figure 3A). This algorithm, which we call Multi-dimensional Integration of Genomic data from Human Tissues (MIGHT), provides a composite three-dimensional graphical output of gene sets demonstrating significant alterations in pair-wise unbiased comparisons across the different array platforms (Figure 3B, 3C). By integrating differences in transcript expression with both methylation status and copy number at a given locus, the MIGHT algorithm not only identifies significant transcriptomic alterations during neoplastic progression, but also elucidates the relative contributions of genomic and epigenetic factors toward such deregulation. The advantage of an integrative approach in developing an accurate "patient-specific" multi-component genetic progression model is illustrated in Figure 4. A chromosome 9p21 hemizygous deletion was identified by aCGH analysis of LGD and HGD biopsies obtained from this individual, which was absent in the matched normal esophageal squamous epithelium, consistent with a somatic monoallelic loss, as confirmed by FISH analysis (Figure $4 \mathrm{~B}$ ). This region harbors two closely approximated tumor suppressor genes: $C D K N 2 A / p 16$ and $C D K N 2 B / p 15$. Prior copy number and other studies have implicated $C D K \mathcal{N} 2 A$ as the target of inactivation at this locus in BE $[15,17,29]$ Nevertheless, in this particular example, microarray data demonstrated that the relative fold reduction in gene expression was considerably greater for $C D K N 2 B(\sim 100$-fold downregulation) than for $C D K N 2 A(\sim 4$ fold); moreover, this finding was independently validated by qRTPCR, which confirmed the complete absence of $C D K N 2 B$ transcripts in the dysplastic biopsy samples, while the expression of $C D K N 2 A$, albeit significantly reduced, was still detectable. Analysis of the third component (HELP analysis) clarified that the $C D K \mathcal{N} 2 B$ promoter in the retained allele underwent progressive hypermethylation during $\mathrm{BE}$ progression, while the $C D K N 2 A$ promoter maintained its methylation status quo (Figure 4A, bottom). This finding suggests the importance of methylation of the remaining allele in regulating expression, and provides direct experimental evidence for genetic and epigenetic hits acting concurrently and synergistically to downregulate tumor suppressor genes during oncogenesis through bi-allelic inactivation (Figure S3).

\section{Integrated multi-platform analysis as a tool for biomarker discovery in multistep carcinogenesis}

Finally, in addition to the value of integrated datasets in understanding mechanisms of transcript disruption (as illustrated above), we explored the utility of the MIGHT algorithm as a tool for biomarker discovery in Barrett progression. In particular, we focused on genes that were significantly overexpressed in pair-wise comparisons, by hypomethylation and / or genomic amplification, and validated selected examples of patient-specific aberrations in larger sample sets.

Using the MIGHT platform, we determined that many genes not previously implicated in esophageal carcinogenesis were significantly upregulated during stepwise progression to cancer (Figure 2 and Tables S1, S2, S3, S4). These were upregulated by either loss of methylation, or gene amplification, or both occurring together. Genes previously known to be important during metaplastic transformation of squamous to columnar epithelium were also included in the list of most significantly upregulated transcripts (for example, villin and mucin genes), confirming the biological validity of our assays. Transcripts corresponding to a family of chemokine ligands were among the most significantly upregulated, and the corresponding gene cluster is present on the 4 q21 chromosomal segment that was amplified in all patient samples during the process of Barrett neoplasia (Figure 5A, 5B) Integrative analysis revealed that in addition to amplification, the CXCL1 and CXCL3 gene promoters were also hypomethylated during transformation, and their relative increase in transcript expression was many fold greater (4-6 fold by array, 10-20 fold by qRT-PCR) than that of $I L-8$, a chemokine gene which was part of the 4 q2 1 amplicon but whose promoter was not affected by loss of methylation (Figure 5A, Bottom). These observations were validated by qRT-PGR in larger independent set of primary samples (Figure 5C), and confirmed a greater than 30-fold mean increase in CXCL1 and CXCL3 levels when compared to $I L-8$ transcripts at each histological grade of $\mathrm{BE}$ progression, thus demonstrating the combinatorial affect of both genetic and 
A

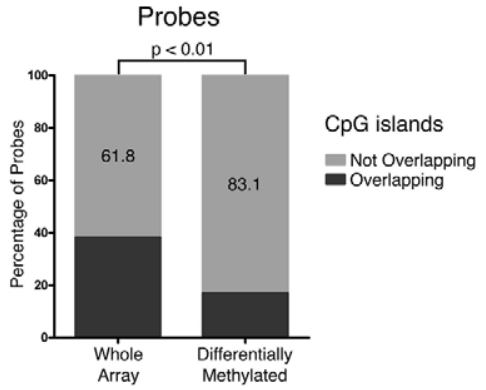

\section{B Chromosome 10}
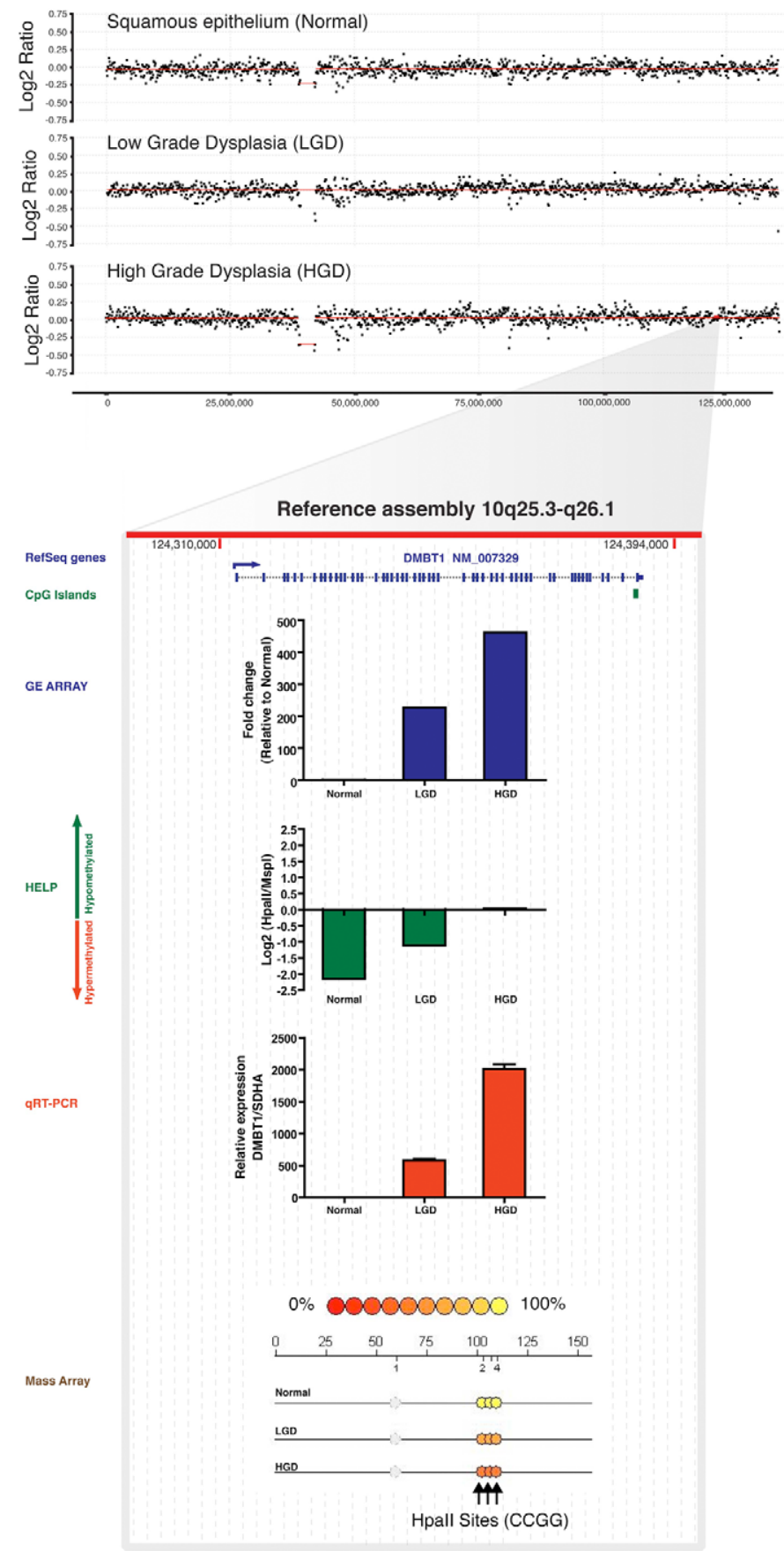

C
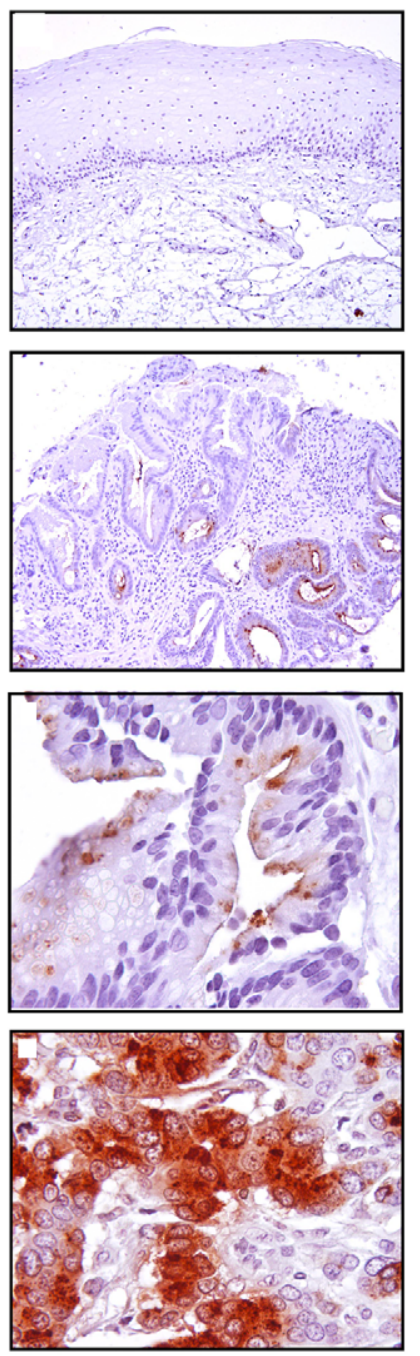

D

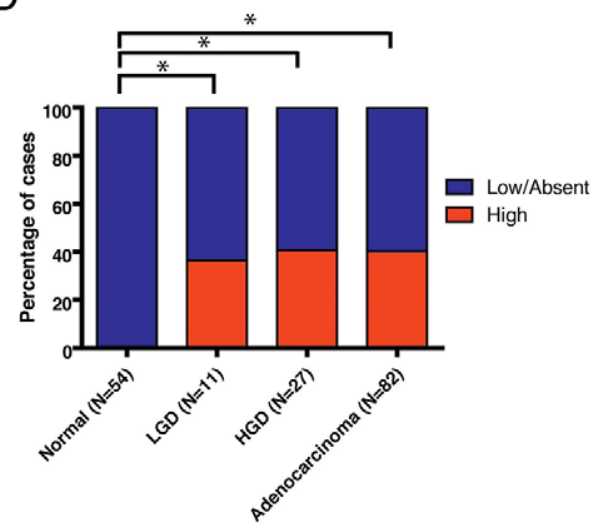


Figure 2. DMBT1 upregulation by non-CpG island methylation changes. Differentially methylated loci between normal esophageal epithelium and Barretts/LGD were mapped to genomic locations of $C p G$ islands. A large percentage of these loci were not within CpG islands. Paired T Test was used to compare to distribution of loci present on the HELP array (A). The DMBT1 gene is not amplified (panel B) and lacks a CpG island in its promoter. DMBT1 was seen to be significantly upregulated and hypomethylated during integrative analysis and marked upregulation was validated by qRT-PCR (red bar). Significant hypomethylation was seen by the HELP assay (green bar) and validated by quantitative MassArray (B). Immunohistochemical evaluation of the protein levels of the DMBT1 gene on primary tissues shows a frequent and significant protein upregulation during early stage dysplasia (panel C,D).

doi:10.1371/journal.pgen.1001356.g002

epigenetic alterations on dysregulation of gene expression during carcinogenesis.

In light of the significant upregulation of chromosome $4 \mathrm{q} 21$ chemokine cluster transcripts in $\mathrm{BE}$ and $\mathrm{EAC}$, and the likely secretion of their protein products into the circulation, we evaluated the potential of using this chemokine familyas serum biomarkers of EAC. Serum samples were collected from an independent cohort of patients with EAC and were compared to samples from patients with gastroesophageal reflux disease (GERD) symptoms without demonstrable BE on histology. Levels of chemokine ligands, IL-8 (IL8), IP-10 (CXCL10), Eotaxin (CCL1 1), MCP-1 (CCL2) and MCP-4 (CCL13) were determined in serum samples by a multiplexed assay on an ultra-sensitive chemiluminiscence detection platform. We observed that both chemokines that were a part of the amplified 4q21 segment (IL-8 and CXCL10) were significantly elevated in the EAC serum samples compared to the controls $(P<0.05)$, while none of chemokines outside of this amplicon demonstrated a significant difference between cancer and control specimens (Figure 5D). These results validate the feasibility of identifying candidate biomarkers by integrative analysis in a limited number of patient samples, and extrapolating their utilization to larger, independent patient cohorts.

To further functionally validate the utility of our integrative discovery platform, we focused on a transcription factor, GATA6, which was significantly overexpressed early in Barrett metaplasia, and was predicted by MIGHT to be amplified without concomitant alterations in methylation (Figure 3). Assessment of the aCGH data and FISH on primary tissues readily validated the copy number alterations at the GATA6 locus during esophageal carcinogenesis (Figure 6A, 6B). Thereafter, using independent cohorts of snap-frozen and archival BE samples, respectively, we observed significant upregulation of GATA6 transcript expression (>500 fold mean increase in LGD, HGD and EAC samples, Figure 6C, 6D) and of the Gata6 protein levels (No Gata6 staining observed in 54 normal controls when compared to mean $60 \%$ positivity in a total of 201 LGD, HGD and EAC samples; Figure $6 \mathrm{~F}-6 \mathrm{I}$ ), confirming the results from the genomic analysis. To validate an oncogenic role in esophageal carcinogenesis, we used an esophageal adenocarcinoma-derived cell line, OE33 [30] and observed significant overexpression of Gata6 protein in these cells (Figure 6J). GATA6 was successfully knocked down using lentiviral short hairpin RNAs in OE33 cells (Figure 6K). Loss of Gata6 function did not affect proliferation, (Figure 6L) but resulted in significantly decreased anchorage independent growth of OE33 cells and also led to decrease in invasion and migration (Figure 6M-6P), thus providing a putative functional association between GATA6 amplification and disease progression in BE.

\section{Discussion}

Esophageal cancer is the cancer with the fastest-growing prevalence in the United States [1] and arises from the metaplastic transformation of normal squamous mucosa, through the intermediate stages of dysplasia, culminating in cancer. Newer insights into the pathogenesis of this process are critically needed for prevention and early diagnosis of these lesions. Although alterations in DNA methylation have been described during esophageal carcinogenesis, studies performed thus far have focused on the aberrant hypermethylation of $\mathrm{CpG}$ islands located within promoters of selected tumor suppressors such as $C D K N 2 A / p 16$, HPP, RUNX3, REPRIMO, amongst others. In contrast, we have
A

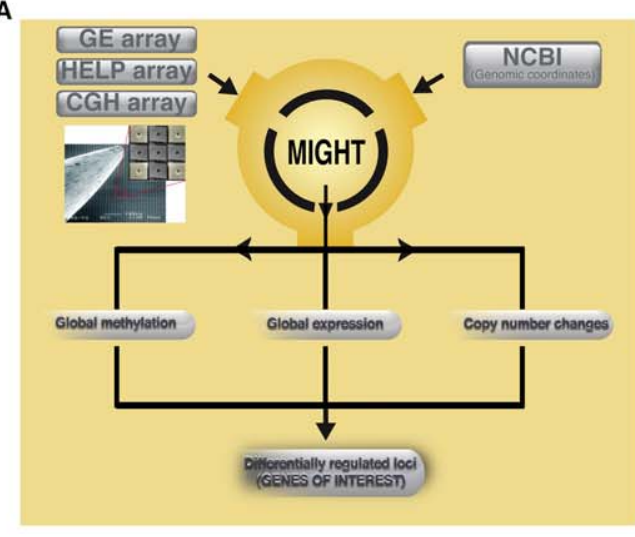

B

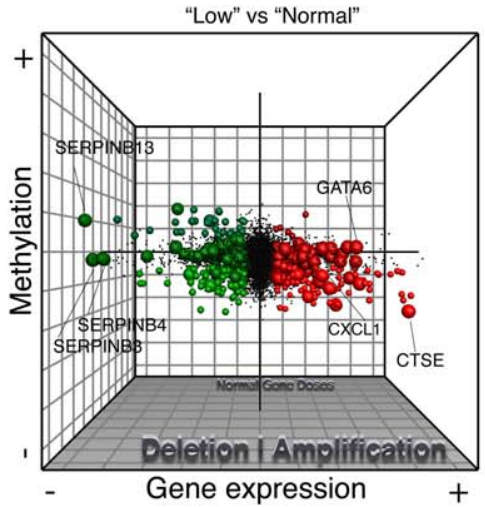

C

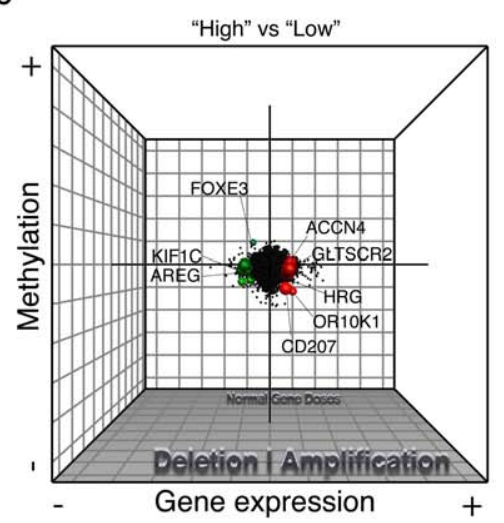

Figure 3. Global integrative analysis of transcriptomic, epigenomic, and DNA copy number data. Probes from all three arrays were linked to genes on the basis of genomic coordinates isolated from NCBI build35. Pairwise analysis was performed between main histologic groups of normal squamous vs LOW (B) and LOW vs HIGH (C). The $x$ axis represents fold-change differences (log2) in gene expression, the $y$ axis represents foldchange differences $(\log 2)$ in methylation and the $z$ axis represents the significance of these changes (-log10 p-value) for both platforms. Upregulated genes ( $>2.5$ fold change in GE with a p-value $<0.05)$ are shown in green and downregulated genes are shown in red. Darker shading of these colors is used to represent genes that are significantly differentially methylated. Hypermethylated genes are at the top of the graph and hypomethylated genes on the bottom. Finally, copy number changes are defined by "volume". Genes with copy number changes (amplified or deleted) are depicted by larger sized circles. This plot allows us to visualize differentially regulated genes and the mechanisms of their dysregulation. doi:10.1371/journal.pgen.1001356.g003 


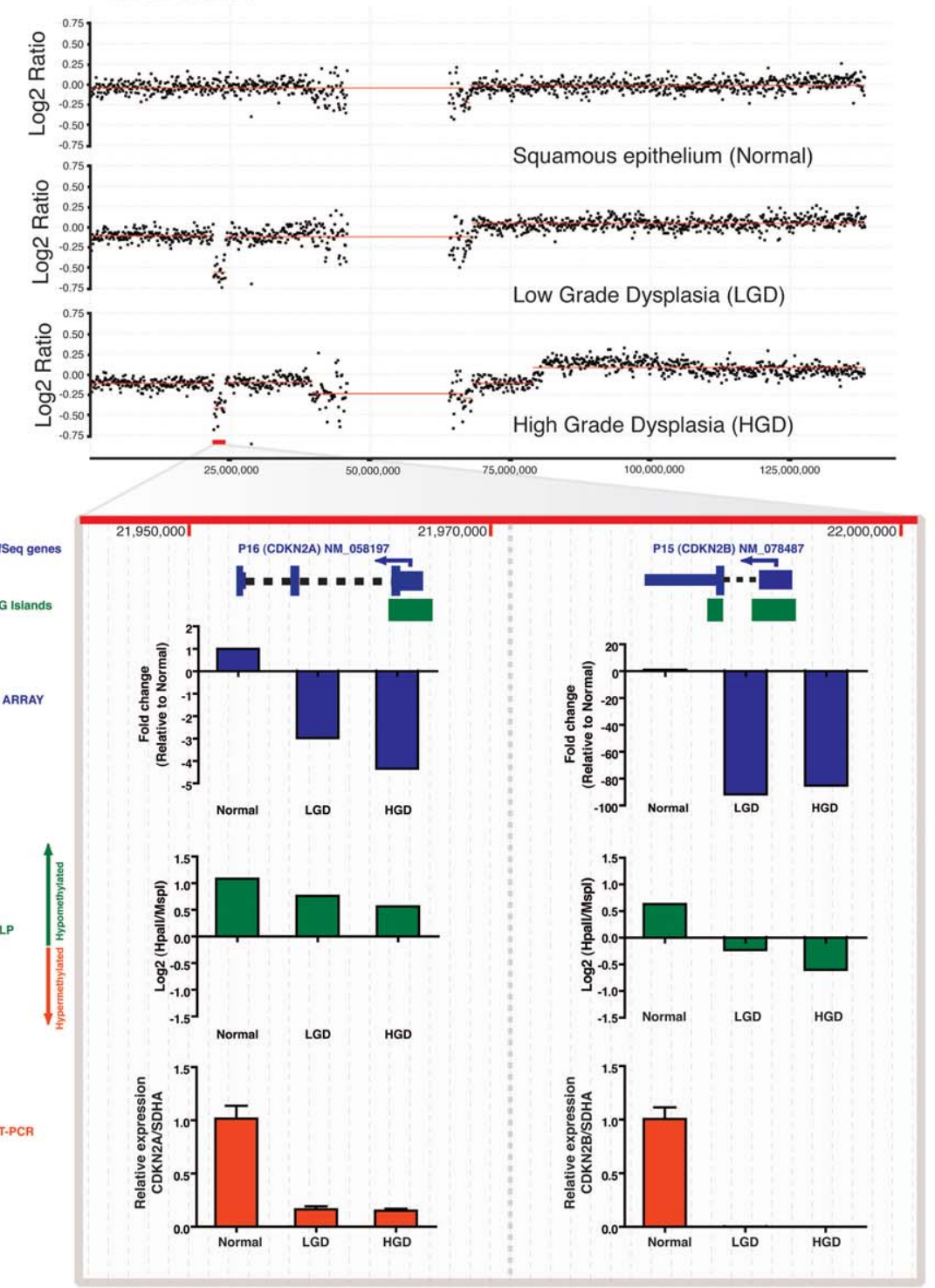

B
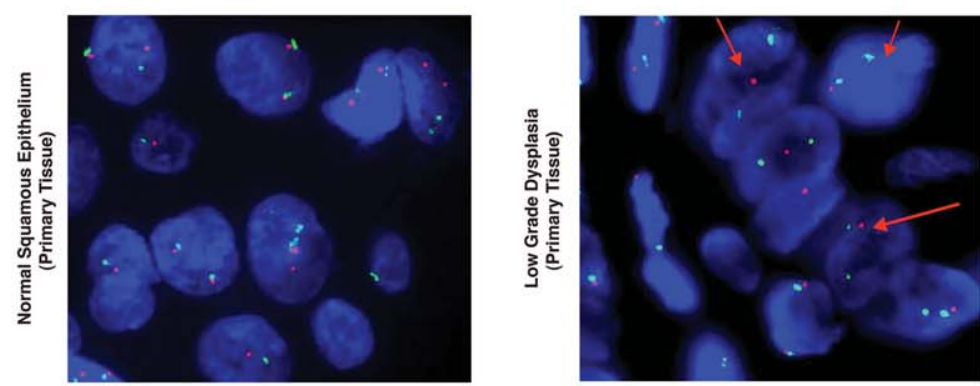

Figure 4. Differential inactivation of the $C D K N 2 A / C D K N 2 B$ locus by combination of genomic and epigenetic mechanisms. In an index patient with serial progression biopsies, there is a hemizygous deletion of the CDKN2A/CDKN2B gene locus at 9p21, beginning at the stage of LGD, and persisting into the corresponding HGD sample (Panel A), which is accompanied by expected downregulation of both transcripts in expression microarray data. Although independent qRT-PCR for CDKN2A and CDKN2B transcripts (red bars) validates the expression array data (blue bars), the magnitude of downregulation of CDKN2B is more pronounced than CDKN2A ( 100-fold versus $\sim 5$-fold on microarray, and complete absence of CDKNA2B transcripts on qRT-PCR versus downregulated but detectable CDKN2A transcripts). MIGHT analysis confirms that this discrepancy between closely spaced hemizygously deleted loci correlates with increased promoter methylation of the residual CDKNA2B allele, as demonstrated by the HELP assay (green bars); such progressive hypomethylation is not observed on the residual CDKN2A allele and likely is responsible for the reduced but demonstrable transcripts. Uniallelic deletion of the CDKN2A/CDKN2B locus is validated by FISH (Panel B) on primary tissue as shown by loss on the LSI p16 SpectrumOrange probe (red arrows). doi:10.1371/journal.pgen.1001356.g004 


\section{Chromosome 4}

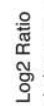

Squamous epithelium (Normal)

54

Low Grade Dysplasia (LGD)

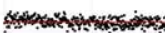

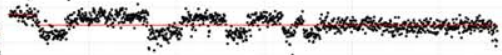

High Grade Dysplasia (HGD)

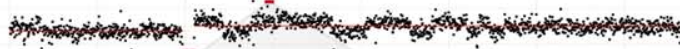

B

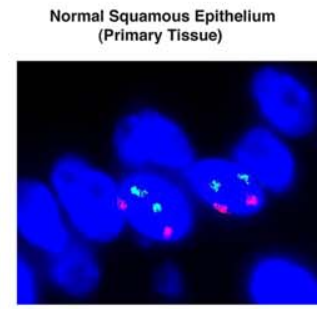

High Grade Dysplasia (Primary Tissue)

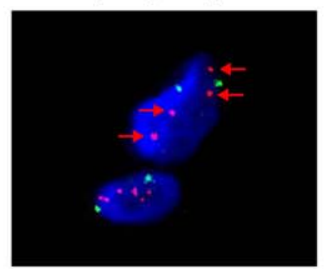

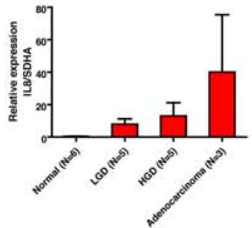
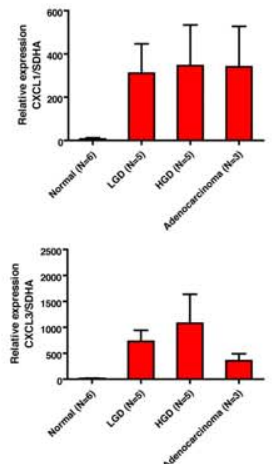

GE ARPAY

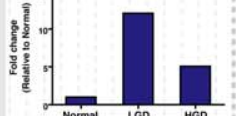

(1)

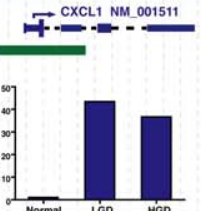

HELP
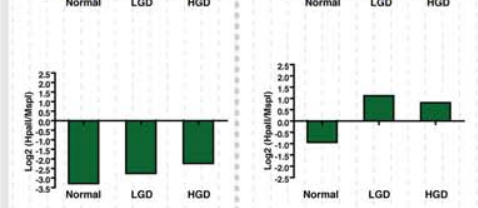

울

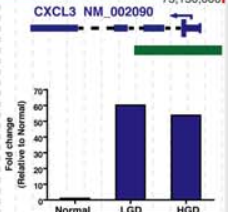

QRT.PCR
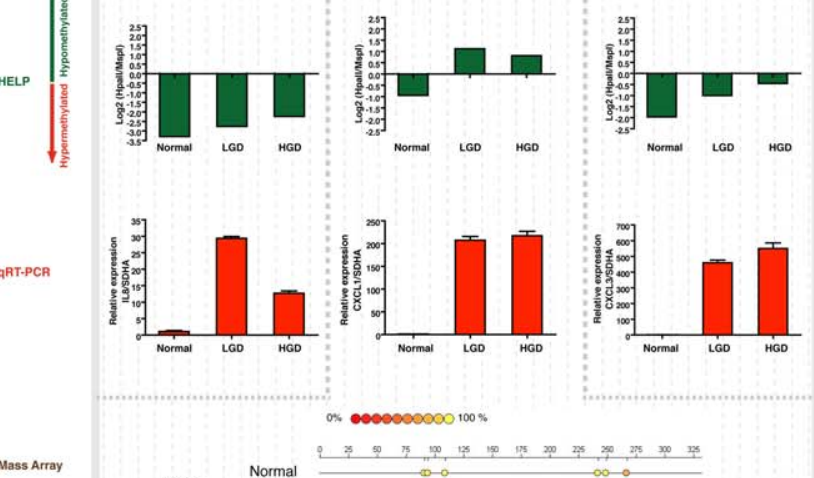

CXCL1

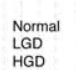

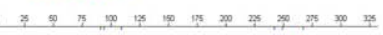

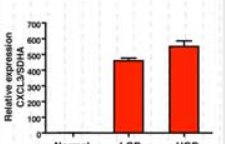

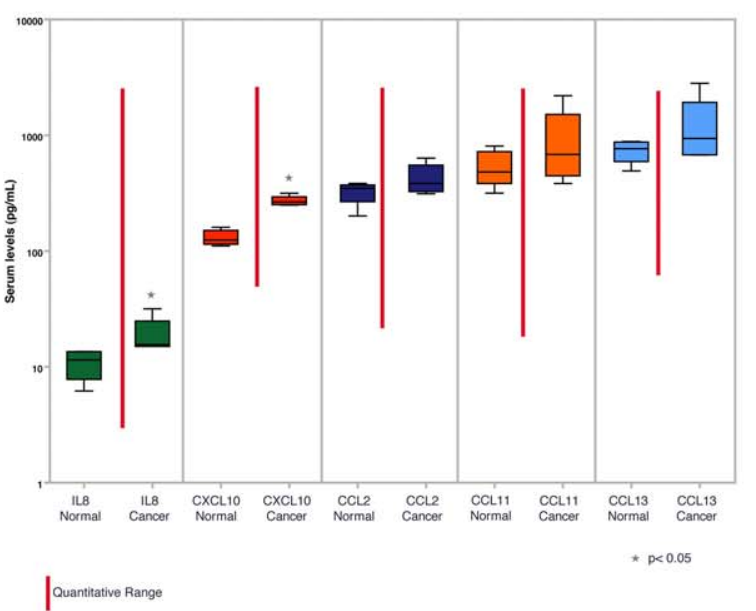

Figure 5. Aberrant expression of a $4 \mathrm{q} 21$ chemokine cluster by gene amplification and promoter hypomethylation during esophageal carcinogenesis. In an index patient with serial progression biopsies, amplification of chromosome $4 \mathrm{q} 21$, containing a cluster of genes transcribing chemokine ligands, is seen beginning at LGD, and persisting into HGD (Panel A). Expression and promoter methylation data for three representative genes in this amplicon (CXCL1, CXCL3, and IL8) are shown below the copy number window. All three transcripts are upregulated in the LGD and HGD samples by microarray data (blue bars), which is validated using independent qRT-PCR assays (red bars). Nonetheless, there is strikingly higher upregulation of CXCL1 (>200-fold on qRT-PCR compared to matched normal) and CXCL3 (500-700 fold on qRT-PCR compared to normal), versus the 10-30 fold upregulation in IL8 mRNA. Integrative analysis on the MIGHT platform confirms that this is likely arises due to progressive hypomethylation of the CXCL1 and CXCL3 promoters (as gauged by HELP assay, green bars), which is not seen at the IL8 promoter. The hypomethylation of $C X C L 1$ observed in the dysplastic samples by the HELP assay is validated by Mass Array at the bottom. The 4q21 chemokine cluster amplification is also confirmed by FISH (Panel B) on the corresponding archival HGD sample from the same individual used in the integrative analysis. The RP11-94K4 SpectrumOrange probe representing the 4q21.1 - 4q21.2 amplicon (red arrows) was used. Moreover, the upregulation of IL8, CXCL1, and CXCL3 transcripts is also confirmed in an independent panel of endoscopic mucosal biopsy samples, including $L G D(N=5), H G D(N=5)$, and adenocarcinoma $(\mathrm{N}=3$ ) (Panel $\mathrm{C})$. Notably, the significantly greater upregulation of $C X C L 1$ and $C X C L 3$ transcripts observed in the index patient persists in this independent cohort of cases. Finally, chemokine detection using a multiplex chemiluminiscent assay was performed on five serum samples from patients with esophageal adenocarcinoma (designated "Cancer") and compared to samples from controls with GERD symptoms and normal esophageal histology (designated "Normal") (Panel D). Serum levels of only two chemokines, IL-8 and CXCL-10, both encoded by the 4q21 cluster, are significantly higher in cancer patients when compared to normals, supporting the evidence that members encoded by this amplicon are expressed at higher levels when compared to chemokines that are not part of the amplicon, such as CCL2, CCL11 and CCL13. Unfortunately, CXCL1 and $C X C L 3$ were not included in this pre-formed multiplex assay.

doi:10.1371/journal.pgen.1001356.g005

determined that hypomethylation, rather than hypermethylation, is the more pervasive epigenetic alteration that occurs during Barrett progression. Additionally, we determined that global cytosine hypomethylation occurs very early during multistep carcinogenesis, observed within the first discernible metaplastic lesions within the native squamous esophagus. Even though global hypomethylation was reported in the pioneering epigenetic studies in cancer [31], most investigators have subsequently focused on hypermethylation in $\mathrm{CpG}$ islands within selected gene promoters. Hypomethylation has been hypothesized to lead to carcinogenesis by encouraging genomic instability [32] as well as by aberrant activation of oncogenes [33]. Additionally, as illustrated in the 


\section{A Chromosome 18}
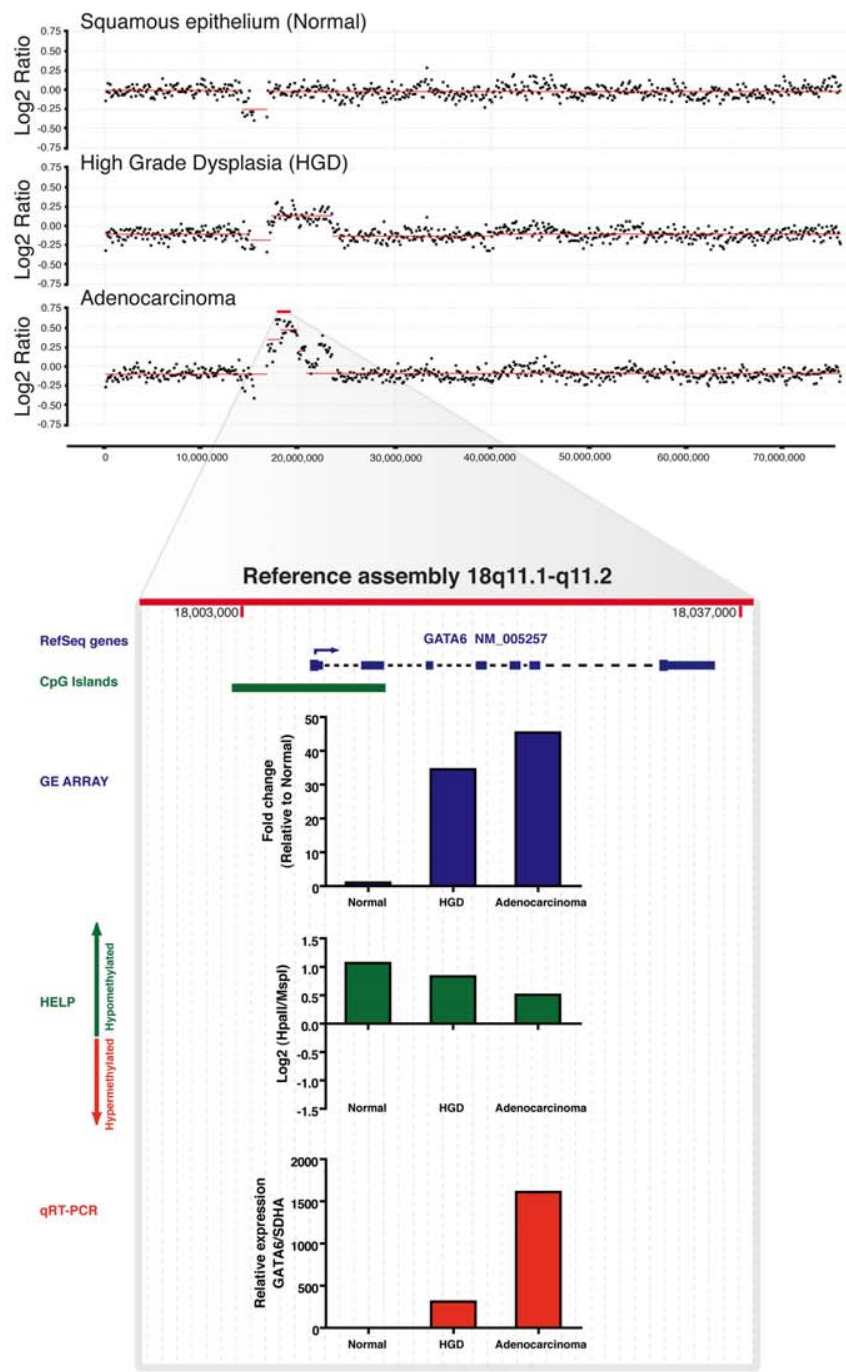

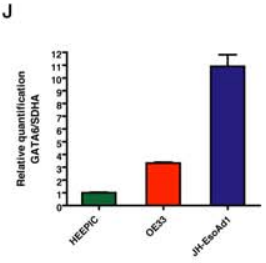

L

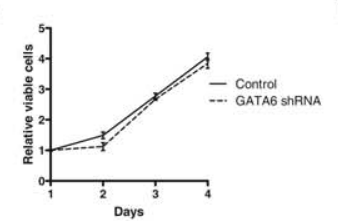

N

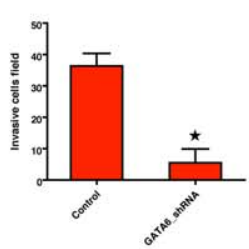

K

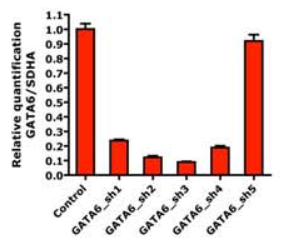

M

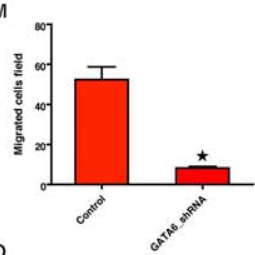

o

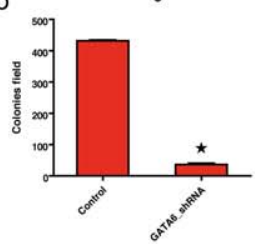

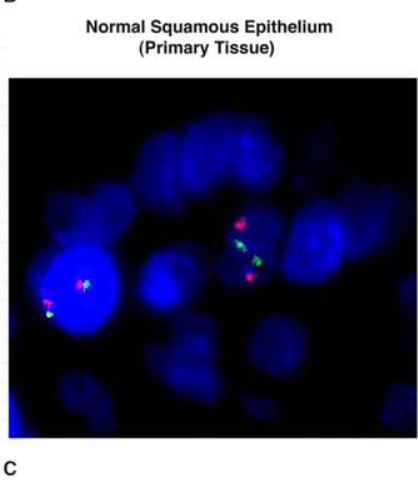
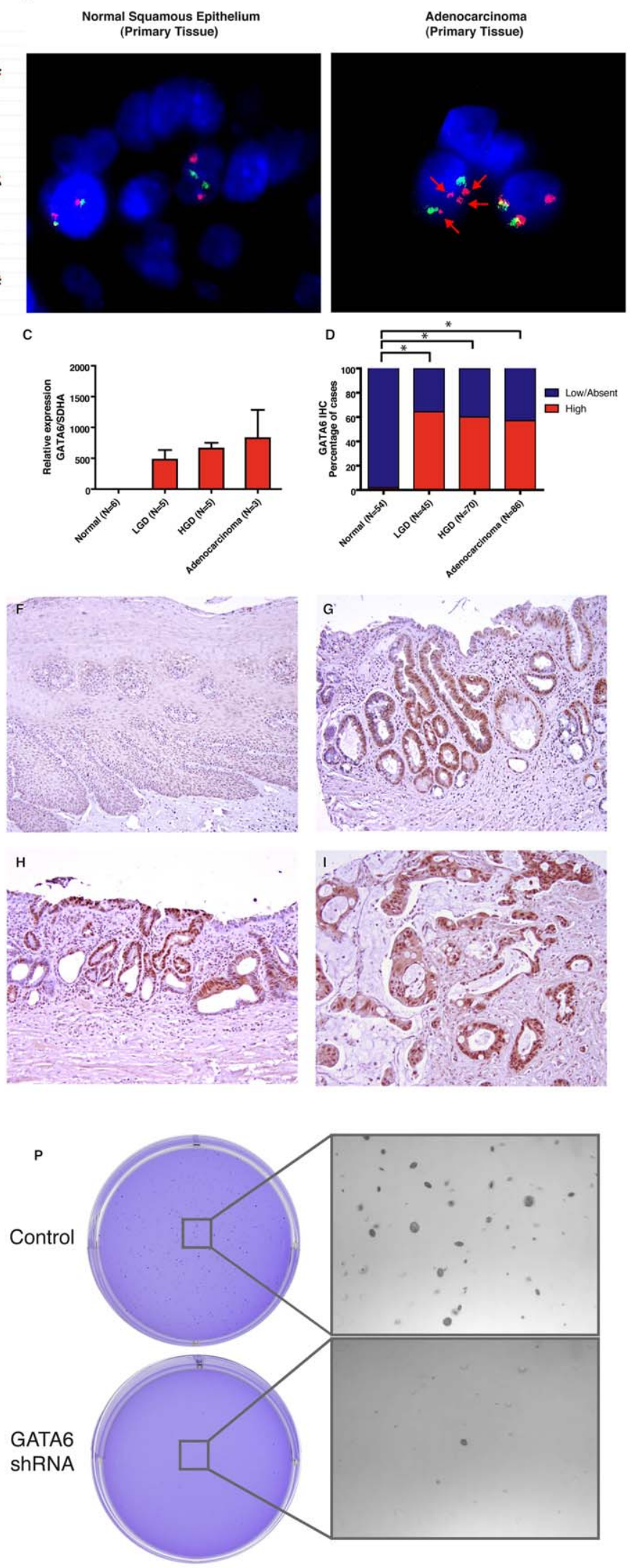
Figure 6. GATA6 is amplified and functions as an oncogene in esophageal adenocarcinoma. In an index patient with serial progression biopsies, aCGH shows a progressive copy number gain on the region 18q11.1-q11.2 from normal to dysplasia to cancer. (Panel A). Integrative approach demonstrates GATA6 and LAMA3 as two genes contained on this cluster (from 17-23,000,000 bp windows) with the marked upregulation during transformation of normal to dysplasia as well as from dysplasia to cancer. qRT-PCR (red bar) validates the findings on the GE array (blue bar) and also correlates with the increasing copy number doses seen in the aCGH. No relevant change is detected by the HELP assay (green bar), showing persistence of unmethylated Hpall sites on the promoter region. GATA6 amplification was corroborated by FISH in the primary adenocarcinoma tissue from this patient (panel B), RP11-18K7 spectrumOrange represent the 18q11.1-q11.2 region containing the GATA6 gene (red arrows). qRT-PCR on independent frozen primary tissues demonstrates high detectable mRNA levels in dysplasia and cancer (Mean $+/-$ SEM). Immunohistochemical analysis on large number of primary tissues demonstrates elevated protein levels arising as early as in the LGD group. Percentage frequency of combined results from TMA and EMR are shown (Panel D) and examples of high IHC scores are shown (Panel F-I). To test the functional role of GATA6 upregulation, its expression was characterized in Barrett's associated adenocarcinoma cell lines (Panel J). Stable knock down of GATA6 from five shRNAs were screened by qRT-PCR in OE33 cell line (Panel K). shRNA-3 was selected for further experiments. Although there is no effect on cell viability (panel L), GATA6 loss significantly decreases cell migration (Panel M), Invasion (Panel N) and anchorage independent growth (panel O-P). doi:10.1371/journal.pgen.1001356.g006

example of DMBT1, hypomethylation alone can lead to transcriptional upregulation during multistep progression to high-grade dysplasia and cancer. Furthermore, our data identify CG dinucelotide loci that can be targeted by differential methylation during neoplastic progression and are located outside of canonical $\mathrm{CpG}$ islands. Importantly, these $\mathrm{CG}$ alterations are not merely stochastic in nature, but appear to have bona fide regulatory influence on transcript expression. Recent work has similarly shown that cytosines present outside of $\mathrm{CpG}$ islands can be aberrantly methylated/ hypomethylated in cancer, and assays that cover these loci are critical to discovering the full landscape of altered methylome of malignancies [34,35].

Since carcinogenesis is multifactorial and gene inactivation and activation can be influenced by either genetic or epigenetic mechanisms, we performed an integrative analysis to dissect the relative contributions of these alterations during this process. Our study provides direct experimental evidence of deletions and promoter methylation acting in concert to silence tumor suppressors in a bi-allelic manner, as first postulated by Knudson's two-hit paradigm. We expand on this paradigm by demonstrating that gene amplifications and hypomethylation can function in concert to upregulate gene expression of various genes. Of note, the use of an integrated approach facilitated by the MIGHT algorithm provides accurate in silico insights into mechanisms of deregulation, particularly when closely spaced gene clusters harbor discrepant alterations in transcript level, as exemplified by $C D K N 2 A$ and $C D K N 2 B$, or the chemokine family in the $4 \mathrm{q} 21$ amplicon. In each of these instances, a subsequent validation step (such as FISH analysis for deletion, or MassArray for promoter methylation, respectively) confirmed the suggested mechanism of transcript deregulation implicated by MIGHT, underscoring the robustness of the analysis platform. Finally, we demonstrate that multiplatform high resolution integrative analysis of limited number of well annotated samples $(\mathrm{N}=$ three patients) can lead to findings that can be extrapolated to larger independent sample cohorts. We have illustrated this paradigm using multiple examples throughout the text, such as with the 4q21 chemokine cluster, DMBT1, and GATA6. In each of these examples, we validated the findings elucidated in the "index" patients in cohorts of either snap frozen or paraffin embedded BE and EAC tissues, In the case of the $4 \mathrm{q} 21$ chemokine cluster, we extended the validation one-step further, using serum samples to confirm significantly elevated circulating levels of two of the cytokines in EAC patients compared to controls. Notably, other chemokine ligands not included within the $4 \mathrm{q} 21$ amplicon failed to demonstrate any significant differences between cancer and control specimens, reiterating the biological relevance of the in silico MIGHT data. Chemokine ligands have recently been shown to be secreted by malignant cells and have been shown to participate in neoplastic progression of melanoma, breast, cervical and colorectal cancers [36-38]. CXCL1, CXCL3 and IL-8 bind to the CXCR2 receptor that has important roles in oncogene-induced senescence. It has been suggested that these chemokines potentiate tumor progression especially with cells with p53 inactivation, an event seen commonly in esophageal neoplasms [39]. These chemokines have also been implicated in tumor associated angiogenesis [37] and are a part of growing evidence of inflammatory mediators implicated in tumor growth and progression [40]. Our data reveals mechanisms associated with their upregulation in EAC and also demonstrates that this upregulation may occur early during neoplastic transformation, potentially allowing the development of a serum-based assay to screen subjects with BE for neoplastic progression.

Overall, our studies suggest that widespread changes in DNA methylation, especially hypomethylation, as well as genomic copy number alterations, can occur early during the multistep process of esophageal carcinogenesis, and may act in concert to deregulate the expression of important potential cancer-related pathogenic genes.

\section{Methods}

\section{Patient samples}

Specimens were obtained from patients who underwent endoscopic surveillance. All patients were diagnosed with Barrett's esophagus. After signed informed consent approved by the Johns Hopkins University IRB, endoscopic biopsies were collected and snap frozen in liquid Nitrogen, de-linked from direct patient identifiers and stored at $-80{ }^{\circ} \mathrm{C}$. DNA and RNA was extracted from the same biopsy samples.

\section{Tissue microarray}

All specimens were obtained from the surgical pathology files of the Johns Hopkins Hospital, Memorial Sloan-Kettering Cancer Center and Karmanos Cancer Center. Tissue microarrays (TMA) were generated from formalin-fixed paraffin-embedded archival tissues from 92 patients with Barrett's Esophagus and included esophageal squamous epithelium (60 cases), low-grade and highgrade dysplasia (19 and 38 cases), and adenocarcinoma (80 cases). Four $1.8 \mathrm{~mm}$ tissue cores represented each case and included two cores from the neoplastic compartment in order to account for potential tumor heterogeneity, and two cores from adjacent normal esophageal parenchyma as an internal control.

Additionally for GATA6 IHC, 120 endoscopic mucosal resection (EMR) specimens from 67 patients with $\mathrm{BE}$ were analyzed, including 31 cases of low grade dysplasia, 40 cases of high grade dysplasia and 10 cases of adenocarcinoma. All specimens were obtained from the surgical pathology files of the Johns Hopkins Hospital. 


\section{Immunohistochemistry}

Rabbit polyclonal GATA6 (H-92) antibody (Santa Cruz Inc, sc9055) was used at 1:500 dilution and visualized using the PowerVision+ Poly-HRP IHC kit (Immunovision Technologies) following the standard protocol for immunohistochemistry (IHC) described previously [41]. Immunohistochemical labeling was assessed in an outcome-blinded fashion by two of the authors (J.C.R and A.M) on a compound microscope. Intensity of labeling was evaluated as previously published $[42,43]$.

\section{FISH analysis}

The UCSG genome browser was used to select for the BAC clones spanning the 18q11.2 region: RP11-18K7 (GATA6, SpectrumOrange); and RP11-49H23 (18q22.2, Control SpectrumGreen). For the CXCL1-3 gene cluster, BAC clones covering the $4 \mathrm{q} 21.1$ - 4q21.2 region were RP11-94K4 (SpectrumOrange) and RP11-259E13 (4q11-q12, Control SpectrumGreen). The BAC clones were obtained from the Children's Hospital Oakland Research Institute in Oakland, USA. We also used commercial LSI p16/CEP probes (Vysis) for spanning the genetic loci for p16 and p15 (SpectrumOrange) and the alpha satellite sequences specific to chromosome 9 (SpectrumGreen).

\section{Cell lines and culture conditions}

Barrett's associated adenocarcinoma cell lines OE33 (European Collection of Cell Cultures, Wiltshire, UK) and JH-EsoAd1, recently described by our group [44], were maintained in RPMI1640 and supplemented with $10 \%$ or $20 \%$ FBS respectively and $100 \mathrm{U} / \mathrm{mL}$ penicillin, $100 \mathrm{mg} / \mathrm{mL}$ streptomycin.

\section{Lentiviral shRNA knockdown of GATA6 in OE33 cells}

GATA6-expressing OE33 cells were seeded into 24-well plates at $9 \times 104$ cells per well concentration, and infected with either scrambled pLKO.1 (18 bp stuffer) lentiviral vector or with lentivirus expressing GATA6 shRNA (Open Biosystems, Huntsville, AL). Stable clones were selected by adding $5 \mu \mathrm{g} / \mathrm{ml}$ of puromycin to the cell culture media. Quantitative reverse transcription PCR (qRT-PCR) analysis was used to select the best short hairpin constructs for GATA6 mRNA knockdown. Downstream experiments were performed with GATA6_sh3 and compared with scrambled control.

\section{Quantitative reverse transcription PCR (qRT-PCR)}

One microgram of total RNA, isolated with the RNAgents kit (Promega, Madison, WI), were reversed transcribed by using the Superscript II First Strand kit (Invitrogen) as per manufacturer's protocol. $1 \mu \mathrm{L}$ of cDNA was amplified in a $25 \mu$ l volume containing $12.5 \mu \mathrm{L}$ of $2 \times$ SYBRGreen PGR Master Mix (Applied Biosystems) and $0.5 \mu \mathrm{M}$ of each primer. Reactions were performed in triplicate using a 7300 Real Time PCR machine (Applied Biosystems, CA, USA) using PCR conditions and data analysis as described earlier[41]. The melting curve was constructed for each primer to ensure reaction specificity. Following PCR, the threshold cycle (CT) was obtained and relative quantities were determined by normalization with the housekeeping gene SDHA.

\section{Statistical analysis}

Data are presented as mean and S.E.M. and were compared using a Student's t-test (or Mann-Whitney U-test, as appropriate). A five-parameter logistic equation was used to calculate the curve fit in the non-linear asymmetric regression. Calculations were done with Graphpad Prims 4.0.

\section{In vitro cell viability assays}

Cell viability assays using the The CellTiter 96AQueousOne Solution Cell Proliferation Assay (Promega, Madison, WI) were performed on control-transfected and shRNA-expressing OE33 cells, as described previously [42]. At each time point evaluation, $20 \mu \mathrm{l} /$ well of the Cell Titer 96 solution was added and incubated for 1 hour. Plates were read on a Wallac-1420 Plate reader at OD of $490 \mathrm{~nm}$ (PerkinElmer, Boston, MA). All experiments were set up in triplicate to determine means and standard deviations.

\section{Boyden chamber migration- and invasion assay}

The Boyden chamber migration- and invasion assays were carried out on OE33 cells. For the invasion assay, $5 \times 104$ cells were suspended in medium containing $0.2 \% \mathrm{FBS}$ and plated in the inner chamber of a matrigel-coated $8-\mu \mathrm{m}$ polypropylene filter inserts (BD Matrigel Matrix, BD Falcon). The bottom chamber contained normal growth media. After $24 \mathrm{~h}$, the cells remaining in the insert were removed with a cotton swab, and the cells on the bottom of the filter were fixed and migrated cells were counted under the microscope. All experiments were set up in triplicate. Boyden chamber migration assay was carried out following the procedure described for the invasion assay except that the cells were plated on uncoated $8-\mu \mathrm{m}$ pore polypropylene filter inserts in the Boyden chambers.

\section{Anchorage-independent growth in soft agar}

Anchorage-independent growth was assessed by colony formation assays in soft-agar, as previously described [41]. Briefly, the soft agar assays were set up in 6-well plates, each well containing a bottom layer of $1 \%$ agarose (Invitrogen), a middle layer of $0.7 \%$ agarose including $5 \times 103$ cells and a top layer comprising of medium only. Subsequently, the plates were kept in a tissue culture incubator maintained at $37^{\circ} \mathrm{C}$ and $5 \% \mathrm{CO}$ for 14 days to allow for colony growth, with top medium being changed on a weekly basis. The assay was terminated at day 14 , when plates were stained with $0.005 \%$ crystal violet (SigmaAldrich) solution. Colony counting was performed for each triplicate condition using an automated ChemiDoc XRS instrument (Bio-Rad, Hercules, CA).

\section{Global Gene expression profiling (GE)}

Total RNA, was isolated using the RNAgents kit (Promega, Madison, WI) as per the manufacturer's instructions and RNA integrity was corroborated with the Bioanalizer 2100. One microgram of RNA was reverse transcribed as published previously [9] and cDNA was submitted to Roche NimbleGen Systems, Inc. (Madison, WI) for labeling and hybridization onto the NimbleGen array (2006-10-26_Human_60mer_1in2) containing at least 10 (60mer) probes designed for 37,364 genes from GenBank build 35. Arrays were scanned using a GenePix 4000B scanner (Axon Instruments) and microarray quality controls were done as previously described [9]. Gene expression microarray data will be submitted to the GEO database for public access. Raw data reports (.pair files) were combined and analyzed with the RMA 7 (Robust Multi-array Analysis) package from NimbleScan 2.3 software, including the algorithm for background correction (data based background correction) and normalization (quantile normalization). After analysis, results of 36,846 unique nimblegene probes are reported as $\log 2$ values. Accession numbers from which NimbleGen probes were designed were linked to GenBank accessions from where Entrez IDs and additional annotations were isolated. 


\section{Global DNA methylation profiling: the HELP assay}

The HELP assay was performed as published previously[23,24], in summary, genomic DNA was isolated from cell lysates $(0.1 \mathrm{M}$ Tris-HCL pH 8.0; 10 mM EDTA pH 8.0 and 1\% SDS), digested overnight at $50 \mathrm{C}$ on Proteinase $\mathrm{K}$, follow by several organic extractions (TE-saturated phenol and Phenol-Chloroform-Isoamyl alcohol), DNA was purified and concentrated by ethanol precipitation and resuspended in LOTE $\mathrm{pH} 7.5$ (3 mM Tris-Hcl and $0.2 \mathrm{mM}$ EDTA). Intact DNA of high molecular weight was corroborated, by electrophoresis on $1 \%$ agarose gel, in all cases. DNA was digested with the restriction enzyme HpaII (methylation-sensitive) and MspI (methylation insensitive). Adapters are ligated to the fragments created by digestion that are subsequently used for ligation-mediated PCR amplification. The two digestion products are differentially labeled with two fluorophores and submitted to Roche NimbleGen Systems, Inc. (Madison, WI) for hybridization onto a human HG17 custom-designed oligonucleotide array (2006-10-26_HG17_HELP_Promoter). The focused array design stand for 25,626 HpaII-amplifiable fragments (HAF) defined by those fragments where two HpaII sites are located 2002000 bp apart with at least some unique sequence between them and selected those located at gene promoters and imprinted regions. Each HAF was represented on the microarray by at least 14 oligonucleotides, each 50 nucleotides in length and randomly distributed across the microarray slide. HELP microarray data will be submitted to the GEO database for public access. Raw data reports (.pair files) went through an analytical pipeline of array performance and quality assessment previously described [45]. 10 and included removal of failed probes, summarization of the remaining probes belonging to the $20 \%$-trimmed mean for each HAF. The functions in this pipeline also allows for quantile normalization that solves the fragment size-dependency of the MspI, HpaII and HpaII/MspI ratio distributions seen in this assay. The $\mathrm{R}$ statistical package (http: //www.r-project.org/) was used to calculate the normalized log2 HpaII/MspI ratios allowing us to semi-quantitatively categorize each loci as methylated or hypomethylated based quantitative methylation detected using MassArray Epityping as a validation approach described below. In all instances, HpaII-amplifiable fragments (HAF) sequence were linked to gene promoters (defined as $2000 \mathrm{bp}$ upstream from transcription start site) by using genomic coordinates from National Center for Biotechnology Information Build 35.

\section{Array Comparative genomic hybridization $(\mathrm{aCGH})$}

DNA was hybridized to a oligonucleotide array CGH (NimbleGen Systems), consisting of 388,560 isothermal probes (length 45-75 bp) covering unique genomic regions (median probe spacing of $6 \mathrm{~kb}$ ). Hybridizations were performed at NimbleGen Facility and a reference sample of Human Male Genomic DNA was used as a normal control. Intensity data underwent through a qspline fit normalization algorithm 11 that was used to compensate for inherent differences in signal between the two dyes. To avoid false positive calls due to local variation in signal intensity, a second pass filter was employed. This filter discards change points if their means are not at least 1.5 SD from each other. Log2-ratio values of the probe signal intensities (Cy3/Cy5) were calculated and 120,000 bp windows-average were generated to visualize copy number changes using the circular binary segmentation algorithm 12 contained on the Roche NimbleScan software. Oligonucleotide array data will be submitted to the GEO database for public access. In all instances, DNA sequence coordinates are from National Center for Biotechnology Information Build 35.

\section{Data integration and 3D visualization}

An analytical pipeline was developed to integrate all platforms. In summary, Gene expression (GE), HELP and aCGH probes were linked to genes base on the basis of genomic coordinates isolated from NCBI build35. Probe to gene links containing data from all three platforms, after proper normalization described above, were considered for 3D integration. TTEST pairwise comparisons of GE and HELP were performed between main histological groups (squamous vs dysplasia and dysplasia vs adenocarcinoma) and fold change differences with their $p$-values were calculated. The last two variables for each pairwise were plotted simultaneously for each gene on a $\mathrm{k}$-space where the $\mathrm{x}$ axis represents fold-change differences $(\log 2)$ in GE and the y axis represents fold-change differences $(\log 2)$ in methylation. The " $z$ " axis is used to represent the significance level in $-\log 10 \mathrm{p}$-value for both platforms. Color-coding for significant cut-offs was defined for those genes over 2.5 fold change in GE with a p-value $<0.05$, with green representing downregulated and red upregulated genes. The same criterion was used to detect significant changes in methylation. A darker shade of color was added to the red and green labels to define the hypermethylated genes (on top of the graph) and lighter shade of red and green was used to define significantly hypomethylated genes.

Copy number alterations were defined by two fold criteria: the gene center approach and the genomic segmentation approach. In the gene center approach, the aCGH probes contained in the intragenic region or promoter region (when intergenic area was not available), were averaged. Computational based approach to find accumulative changes on $50 \%$ of DNA content in all three histological stages for each patient was considered gain or loss depending if the progressive trend was positive or negative respectively. A similar approach was also taken for the Circular binary segmentation (CBS) analysis, this time visual inspection at genomic windows of $120,000 \mathrm{bp}$ was applied and genomic segments isolated and classified as gain or losses. Genomic windows were linked to genes and gene copy number changes for each patient was defined as those that agreed on last two criteria. Finally, on the integrative figure, copy number changes were depicted by larger circles. Genes classified as amplified and deleted had a 3 fold more larger size compared to their normal counterparts.

\section{Quantitative DNA methylation analysis by MassArray Epityping}

Quantitative high-throughput DNA methylation analysis to validate HELP results was carried out by MALDI-TOF mass spectrometry using Sequenom's EpiTYPER platform (Sequenom. San Diego, CA). The same DNA aliquots used for HELP were bisulfite-converted; PCR amplified and follow by an in vitro RNA transcription and a base specific cleavage. MALDI TOF MS analysis of the cleavage product was performed as described originally [24,25]. MassArray primers were designed and are available upon request.

\section{Chemokine and cytokine detection}

Five serum samples from patients with gastroesophageal reflux disease (GERD) symptoms and normal esophageal histology, and five serum samples from esophageal adenocarcinoma patients were collected for analysis. Frozen endoscopic tissues from the same patients were also collected and used for tissue lysates. To determine cytokine levels in the serum, Meso Scale Discovery System multiplex assay were used for human Eotaxin (CCL11), 
IL-8 (IL8), IP-10 (CXCL10), MCP-1 (CGL2) and MCP-4 (CCL13) according to the manufacturer's instructions.

\section{Luminometric methylation assay (LUMA)}

Genomic DNA (200-500 ng) was cleaved with HpaII + EcoRI or $\mathrm{MspI}+\mathrm{EcoRI}$ in two separate $20 \mu \mathrm{l}$ reactions containing $33 \mathrm{mM}$ Tris-acetate, $10 \mathrm{mM} \mathrm{Mg}$-acetate, $66 \mathrm{mM} \mathrm{K}$-acetate $\mathrm{pH}$ $7.9,0.1 \mathrm{mg} / \mathrm{ml} \mathrm{BSA}$ and 5 units of each restriction enzymes. The reactions were set up in a 96-well format and incubated at $37^{\circ} \mathrm{C}$ for $4 \mathrm{~h}$. Then $20 \mu \mathrm{l}$ annealing buffer $(20 \mathrm{mM}$ Tris-acetate, $2 \mathrm{mM}$ $\mathrm{Mg}$-acetate $\mathrm{pH}$ 7.6) was added to the cleavage reactions, and samples were placed in a PSQ96 ${ }^{\mathrm{TM}} \mathrm{MA}$ system (Biotage $\mathrm{AB}$, Uppsala, Sweden). The instrument was programmed to add dNTPs in four consecutive steps including Step 1: dATP (the derivative $\mathrm{dATP} \alpha \mathrm{S}$ is used since it will not react directly with Luciferase and prevents non-specific signals); Step 2: mixture of dGTP + dCTP; Step 3: dTTP; and Step 4: mixture of dGTP + dCTP. Peak heights were calculated using the PSQ96 ${ }^{\mathrm{TM}} \mathrm{MA}$ software. The HpaII/EcoRI and MspI/EcoRI ratios were calculated as $(\mathrm{dGTP}+\mathrm{dCTP}) / \mathrm{dATP}$ for the respective reactions. The HpaII/MspI ratio was defined as (HpaII/EcoRI)/(MspI/ EcoRI) [46].

\section{Supporting Information}

Figure S1 Location of Esophageal Samples: Endoscopic biopsies from 3 patients (depicted with different colors) were taken. Green circles represent a 87 yr old male, Blue circles represent a 77 yr old male and Orange circle represent a 56 yr old female. $\mathrm{N}=$ Normal esophagus; B = Barrett's esophagus; L = LGD, H = HGD; A = Adenocarcinoma.

Found at: doi:10.1371/journal.pgen.1001356.s001 (0.10 MB DOC)

Figure S2 Methylation data obtained from the HELP assay correlates with MassArray Epityper analysis.

Found at: doi:10.1371/journal.pgen.1001356.s002 (0.19 MB DOC)

Figure S3 Schematic representation of combined effects of genetic and epigenetic events in esophageal: The first panel shows that uniallelic genetic deletion of the chr9p21 locus in dysplasia results in downregulation of both CDKN2A/p16 and CDKN2B/ p15 genes. The reduction in expression though is much greater for p15 as the promoter gets hypermethylated. The second panel shows that in addition to amplification, the CXCL1 and CXCL3 gene promoters were also hypomethylated during transformation, and their relative increase in transcript expression was many fold greater ( $4-6$ fold by array, 10-20 fold by qRT-PCR) than that of IL-8, a chemokine gene which was part of the 4q21 amplicon but whose promoter was not affected by loss of methylation.

Found at: doi:10.1371/journal.pgen.1001356.s003 (0.05 MB DOG)

Table S1 Genes overexpressed and amplified during transformation of normal esophageal squamous epithelium to Barrett's esophagus and low grade dysplasia.

Found at: doi:10.1371/journal.pgen.1001356.s004 (0.14 MB DOC)

Table S2 Genes overexpressed and hypomethylated during transformation of normal esophageal squamous epithelium to Barett's esophagus and low grade dysplasia.

Found at: doi:10.1371/journal.pgen.1001356.s005 (0.13 MB DOC)

Table S3 Genes overexpressed, amplified and hypomethylated during transformation of normal esophageal squamous epithelium to Barett's esophagus and low grade dysplasia.

Found at: doi:10.1371/journal.pgen.1001356.s006 (0.04 MB DOC)

Table S4 Genes significantly hypomethylated during transformation with methylation changes occurring outside of $\mathrm{CpG}$ islands.

Found at: doi:10.1371/journal.pgen.1001356.s007 (0.09 MB DOC)

\section{Author Contributions}

Conceived and designed the experiments: A Verma, JC Roa, Y Mo, KH Ramesh, A Maitra. Performed the experiments: H Alvarez, J Opalinska, L Zhou, C Montagna, T Bhagat, L Cannizzaro. Analyzed the data: H Alvarez, D Sohal, MJ Fazzari, Y Yu, RF Thompson, M Suzuki, SJ Meltzer, A Melnick. Contributed reagents/materials/analysis tools: EA Montgomery, M Canto, KB Dunbar, J Wang, J Mollenhauer. Wrote the paper: A Verma, A Maitra.

\section{References}

1. Zhang HY, Spechler SJ, Souza RF (2009) Esophageal adenocarcinoma arising in Barrett esophagus. Cancer Lett 275: 170-177.

2. Eloubeidi MA, Mason AC, Desmond RA, El-Serag HB (2003) Temporal trends (1973-1997) in survival of patients with esophageal adenocarcinoma in the United States: a glimmer of hope? Am J Gastroenterol 98: 1627-1633.

3. Montgomery E, Bronner MP, Goldblum JR, Greenson JK, Haber MM, et al. (2001) Reproducibility of the diagnosis of dysplasia in Barrett esophagus: a reaffirmation. Hum Pathol 32: 368-378.

4. Montgomery E, Goldblum JR, Greenson JK, Haber MM, Lamps LW, et al. (2001) Dysplasia as a predictive marker for invasive carcinoma in Barrett esophagus: a follow-up study based on 138 cases from a diagnostic variability study. Hum Pathol 32: 379-388.

5. Spechler SJ (2002) Clinical practice. Barrett's Esophagus. N Engl J Med 346: 836-842.

6. Shaheen NJ, Sharma P, Overholt BF, Wolfsen HC, Sampliner RE, et al. (2009) Radiofrequency ablation in Barrett's esophagus with dysplasia. N Engl J Med 360: 2277-2288

7. Wang S, Zhan M, Yin J, Abraham JM, Mori Y, et al. (2006) Transcriptional profiling suggests that Barrett's metaplasia is an early intermediate stage in esophageal adenocarcinogenesis. Oncogene 25: 3346-3356.

8. Razvi MH, Peng D, Dar AA, Powell SM, Frierson HF, Jr., et al. (2007) Transcriptional oncogenomic hot spots in Barrett's adenocarcinomas: serial analysis of gene expression. Genes Chromosomes Cancer 46: 914-928.

9. Selaru FM, Zou T, Xu Y, Shustova V, Yin J, et al. (2002) Global gene expression profiling in Barrett's esophagus and esophageal cancer: a comparative analysis using cDNA microarrays. Oncogene 21: 475-478.

10. Xu Y, Selaru FM, Yin J, Zou TT, Shustova V, et al, (2002) Artificial neural networks and gene filtering distinguish between global gene expression profiles of Barrett's esophagus and esophageal cancer. Cancer Res 62: 3493-3497.

11. Powell SM, Papadopoulos N, Kinzler KW, Smolinski KN, Meltzer SJ (1994) APC gene mutations in the mutation cluster region are rare in esophageal cancers. Gastroenterology 107: 1759-1763.

12. Meltzer SJ, Yin J, Huang Y, McDaniel TK, Newkirk C, et al. (1991) Reduction to homozygosity involving p53 in esophageal cancers demonstrated by the polymerase chain reaction. Proc Natl Acad Sci U S A 88: 4976-4980.

13. Huang Y, Boynton RF, Blount PL, Silverstein RJ, Yin J, et al. (1992) Loss of heterozygosity involves multiple tumor suppressor genes in human esophageal cancers. Cancer Res 52: 6525-6530.

14. Jin Z, Mori Y, Yang J, Sato F, Ito T, et al. (2007) Hypermethylation of the nellike 1 gene is a common and early event and is associated with poor prognosis in early-stage esophageal adenocarcinoma. Oncogene 26: 6332-6340.

15. Lai LA, Paulson TG, Li X, Sanchez CA, Maley C, et al. (2007) Increasing genomic instability during premalignant neoplastic progression revealed through high resolution array-CGH. Genes Chromosomes Cancer 46: 532-542.

16. Powell EL, Leoni LM, Canto MI, Forastiere AA, Iocobuzio-Donahue CA, et al. (2005) Concordant loss of MTAP and p16/CDKN2A expression in gastroesophageal carcinogenesis: evidence of homozygous deletion in esophageal noninvasive precursor lesions and therapeutic implications. Am J Surg Pathol 29: $1497-1504$.

17. Schulmann K, Sterian A, Berki A, Yin J, Sato F, et al. (2005) Inactivation of p16, RUNX3, and HPP1 occurs early in Barrett's-associated neoplastic progression and predicts progression risk. Oncogene 24: 4138-4148. 
18. Jin Z, Cheng Y, Gu W, Zheng Y, Sato F, et al. (2009) A multicenter, doubleblinded validation study of methylation biomarkers for progression prediction in Barrett's esophagus. Cancer Res 69: 4112-4115.

19. Sato F, Jin Z, Schulmann K, Wang J, Greenwald BD, et al. (2008) Three-tiered risk stratification model to predict progression in Barrett's esophagus using epigenetic and clinical features. PLoS ONE 3: e1890. doi:10.1371/journal. pone.0001890.

20. Prasad GA, Wang KK, Halling KC, Buttar NS, Wongkeesong LM, et al. (2008) Utility of biomarkers in prediction of response to ablative therapy in Barrett's esophagus. Gastroenterology 135: 370-379.

21. Figueroa ME, Reimers M, Thompson RF, Ye K, Li Y, et al. (2008) An integrative genomic and epigenomic approach for the study of transcriptional regulation. PLoS ONE 3: e1882. doi:10.1371/journal.pone.0001882.

22. Leary RJ, Lin JC, Cummins J, Boca S, Wood LD, et al. (2008) Integrated analysis of homozygous deletions, focal amplifications, and sequence alterations in breast and colorectal cancers. Proc Natl Acad Sci U S A 105: 16224-16229.

23. Khulan B, Thompson RF, Ye K, Fazzari MJ, Suzuki M, et al. (2006) Comparative isoschizomer profiling of cytosine methylation: the HELP assay. Genome Res 16: 1046-1055.

24. Oda M, Glass JL, Thompson RF, Mo Y, Olivier EN, et al. (2009) Highresolution genome-wide cytosine methylation profiling with simultaneous copy number analysis and optimization for limited cell numbers. Nucleic Acids Res 37: 3829-3839.

25. Figueroa ME, Lugthart S, Li Y, Erpelinck-Verschueren C, Deng X, et al. DNA Methylation Signatures Identify Biologically Distinct Subtypes in Acute Myeloid Leukemia. Cancer Cell 17: 13-27.

26. Baylin SB, Herman JG, Graff JR, Vertino PM, Issa JP (1998) Alterations in DNA methylation: a fundamental aspect of neoplasia. Adv Cancer Res 72: 141-196.

27. Toyota M, Issa JP (1999) CpG island methylator phenotypes in aging and cancer. Semin Cancer Biol 9: 349-357.

28. Fazzari MJ, Greally JM (2004) Epigenomics: beyond CpG islands. Nat Rev Genet 5: 446-455.

29. Albrecht B, Hausmann M, Zitzelsberger H, Stein H, Siewert JR, et al. (2004) Array-based comparative genomic hybridization for the detection of DNA sequence copy number changes in Barrett's adenocarcinoma. J Pathol 203: 780-788.

30. Abdalla SI, Sanderson IR, Fitzgerald RC (2005) Effect of inflammation on cyclooxygenase (COX)-2 expression in benign and malignant oesophageal cells. Carcinogenesis 26: 1627-1633.

31. Feinberg AP, Vogelstein B (1983) Hypomethylation distinguishes genes of some human cancers from their normal counterparts. Nature 301: 89-92.
32. Rodriguez J, Frigola J, Vendrell E, Risques RA, Fraga MF, et al. (2006) Chromosomal instability correlates with genome-wide DNA demethylation in human primary colorectal cancers. Cancer Res 66: 8462-9468.

33. Ehrlich M (2002) DNA methylation in cancer: too much, but also too little. Oncogene 21: 5400-5413.

34. Doi A, Park IH, Wen B, Murakami P, Aryee MJ, et al. (2009) Differential methylation of tissue- and cancer-specific $\mathrm{CpG}$ island shores distinguishes human induced pluripotent stem cells, embryonic stem cells and fibroblasts. Nat Genet 41: 1350-1353.

35. Figueroa ME, Skrabanek L, Li Y, Jiemjit A, Fandy TE, et al. (2009) MDS and secondary AML display unique patterns and abundance of aberrant DNA methylation. Blood 114: 3448-3458.

36. Sparmann A, Bar-Sagi D (2004) Ras-induced interleukin-8 expression plays a critical role in tumor growth and angiogenesis. Cancer Cell 6: 447-458.

37. Wang D, Wang H, Brown J, Daikoku T, Ning W, et al. (2006) CXCL1 induced by prostaglandin E2 promotes angiogenesis in colorectal cancer. J Exp Med 203: 941-951.

38. Dhawan P, Richmond A (2002) Role of CXCL1 in tumorigenesis of melanoma. J Leukoc Biol 72: 9-18.

39. Acosta JC, O'Loghlen A, Banito A, Guijarro MV, Augert A, et al. (2008) Chemokine signaling via the CXCR2 receptor reinforces senescence. Cell 133: 1006-1018.

40. Coussens LM, Werb Z (2002) Inflammation and cancer. Nature 420: 860-867.

41. Koorstra JB, Karikari CA, Feldmann G, Bisht S, Rojas PL, et al. (2009) The Axl receptor tyrosine kinase confers an adverse prognostic influence in pancreatic cancer and represents a new therapeutic target. Cancer Biol Ther 8: 618-626.

42. Hansel DE, Dhara S, Huang RC, Ashfaq R, Deasel M, et al. (2005) CDC2/ CDK1 Expression in Esophageal Adenocarcinoma and Precursor Lesions Serves as a Diagnostic and Cancer Progression Marker and Potential Novel Drug Target. Am J Surg Pathol 29: 390-399.

43. Montgomery E, Mamelak AJ, Gibson M, Maitra A, Sheikh S, et al. (2006) Overexpression of claudin proteins in esophageal adenocarcinoma and its precursor lesions. Appl Immunohistochem Mol Morphol 14: 24-30.

44. Alvarez H, Koorstra JB, Hong SM, Boonstra JJ, Dinjens WN, et al. (2008) Establishment and characterization of a bona fide barrett esophagus-associated adenocarcinoma cell line. Cancer Biol Ther 7.

45. Thompson RF, Suzuki M, Lau KW, Greally JM (2009) A pipeline for the quantitative analysis of CG dinucleotide methylation using mass spectrometry. Bioinformatics 25: 2164-2170.

46. Karimi M, Johansson S, Stach D, Corcoran M, Grander D, et al. (2006) LUMA (LUminometric Methylation Assay)--a high throughput method to the analysis of genomic DNA methylation. Exp Cell Res 312: 1989-1995. 